\title{
Panax ginseng therapy for chronic obstructive pulmonary disease: a clinical trial protocol and pilot study
}

\author{
Lei Wu', Anthony Lin Zhang ${ }^{2}$, Yuan Ming Di², Johannah Linda Shergis², Yuanbin Chen ${ }^{1}$, Xinfeng Guo',
} Zehuai Wen ${ }^{1}$, Francis Thien ${ }^{3}$, Christopher Worsnop ${ }^{4}$, Lin Lin ${ }^{1 *}$ and Charlie Changli Xue ${ }^{2^{*}}$

\begin{abstract}
Background: Panax ginseng (Ren shen) has been used to treat chronic obstructive pulmonary disease (COPD). This article aims to present a study protocol and pilot trial comparing $P$. ginseng with placebo for treating moderate to very severe COPD.

Methods: COPD was diagnosed spirometrically, with participants having a forced expiratory volume in one second (FEV1) of between 20\% and 79\% and FEV1 to forced vital capacity (FVC) ratio of less than 70\%. Outcome measures included exacerbation rate, St. Georges Respiratory Questionnaire, COPD Assessment Test and Short-form Health Survey (SF-36). Other outcome measures included the six-minute walk test, FEV1, FVC, relief medication use, use of COPD-specific medical resources, and adverse events. The study is a randomized, double-blind, placebo controlled clinical trial. The method of this pilot trial was based on a planned full-scale trial except that participants were enrolled for ten weeks compared to 52 weeks. In the pilot trial, 14 participants (57-73 years old) with moderate to very severe COPD were recruited from a community health program at a public Chinese medicine hospital in Guangdong Province, China. After a 2-week run-in period, 10 participants were eligible for the study and were randomly assigned to either $P$. ginseng group $(n=5)$ (200 mg twice daily for four weeks) or placebo group $(n=5)$, and then followed-up for an additional 4 weeks for a total of 10 weeks.
\end{abstract}

Results: Nine participants completed the trial and one dropped out. The exacerbation rate could not be evaluated because there were no exacerbations. One participant in P. ginseng group reported events of sore throat, cough and fever. Trial investigators did not consider these events as COPD exacerbations or adverse events.

Conclusions: Participant recruitment, study design, data collection and outcome measurement have been tested in a pilot trial. A full-scale trial is warranted.

\section{Background}

Chronic obstructive pulmonary disease (COPD) is characterized by breathlessness, coughing, and excess sputum production [1]. There are 64 million people suffering from COPD worldwide and COPD is predicted to be the third leading cause of death by 2030 [2].

\footnotetext{
* Correspondence: drlinlin620@163.com; charlie.xue@rmit.edu.au 'Guangdong Provincial Hospital of Chinese Medicine, Guangdong 510120, China

${ }^{2}$ Traditional and Complementary Medicine Program, School of Health Sciences and Health Innovations Research Institute (HIRi), RMIT University, PO Box 71, Bundoora, Victoria 3083, Australia

Full list of author information is available at the end of the article
}

COPD patients often use inhaled bronchodilators to relieve symptoms, and inhaled glucocorticoids to treat and prevent frequent exacerbations [1]. New treatments targeting the underlying chronic inflammatory pathways are being developed [3]. A phosphodiesterase-4 (PDE-4) inhibitor, roflumilast (trade names Daxas/Dalisresp), is used to treat COPD, although it has adverse effects, such as nausea, reduced appetite and abdominal pain [1].

Several systematic reviews indicated $P$. ginseng improved lung function (forced expiratory volume in one second, FEV1) and quality of life measured by the St. George's Respiratory Questionnaire (SGRQ), compared to pharmacotherapy alone $[4,5]$. Studies investigating $P$. ginseng for COPD and other related diseases such as 
chronic bronchitis are limited but promising [6-8]. Gross and colleagues demonstrated that $P$. ginseng (G115, $100 \mathrm{mg}$ ) twice daily for 12 weeks improved pulmonary function tests, and respiratory endurance in 92 moderate COPD participants [6]. Scaglione and colleagues evaluated $P$. ginseng in two studies. The first involved 40 chronic bronchitis participants and found that $P$. ginseng $(100 \mathrm{mg})$ twice daily for 8 weeks had fewer alveolar macrophages in bronchoalveolar lavage fluid than those who took placebo [7]. The second study involved 75 participants with acute exacerbation of chronic bronchitis and found that those who took $P$. ginseng $(\mathrm{G} 115,100 \mathrm{mg})$ twice daily with antibiotics for 9 days had a lower bacterial count than those who took antibiotics alone [8]. In addition, no severe side effect was reported in the $P$. ginseng treatment [9]. Despite positive results, these studies did not evaluate appropriate COPD outcomes, such as health-related quality of life and rates of exacerbation.

We previously designed randomized clinical trials (RCT) to evaluate $P$. ginseng's effectiveness in treating moderate COPD [10] and identified methods to integrate Chinese medicine in hospital-based clinical trials [11]. This study aims to present the protocol and a pilot trial comparing $P$. ginseng with placebo for moderate to very severe COPD participants.

\section{Method}

\section{Participants}

We included participants who were aged between 40 and 80 years; had a post-bronchodilator FEV1 of $\leq 20 \%$ and $<80 \%$ of predicted normal values and had a FEV1 to forced vital capacity (FVC) ratio (FER) of less than 70\%; were clinically stable and had not experienced an acute exacerbation of COPD for at least 4 weeks before the trial and had not been hospitalised in the past six months with three or more exacerbations; and met the Chinese medicine diagnostic criteria for lung qi deficiency with or without spleen or kidney qi deficiency.

We excluded participants who had a history of asthma or chronic systemic infections or inflammatory conditions other than COPD that require systemic corticosteroid treatment in the last 3 months; were pregnant, breast-feeding or intending to become pregnant during the course of the study; had a serious illness such as severe heart, liver or kidney disease; were taking long-term immunosuppressive agents or immune-stimulants; had an allergic history to ginseng or currently were taking ginseng; were users of monoamine oxidase inhibitor antidepressants, anticoagulants and/or antihyperglycaemic medications; and had undertaken pulmonary rehabilitation within three months of the commencement of the study or intended to enter pulmonary rehabilitation during the study.

\section{Design}

This pilot trial was designed to test the practicality of a full-scale trial that was planned to be conducted in a public hospital in China. The only difference between the pilot trial and the full-scale trial is the trial duration, being 10 weeks for the pilot and one year for the full scale trial.

The participants with moderate, severe or very severe COPD (Global Initiative for Chronic Obstructive Lung Disease [GOLD], stage II-IV) [1] were recruited from the Guangdong Provincial Hospital of Chinese Medicine, Guangdong Province, China. The participants who provided informed consent were enrolled for 10 weeks: 2 weeks for run-in; 4 weeks for treatment; and 4 weeks for follow-up. The participants meeting all the criteria entered a 2-week run in period. They were randomized to $P$. ginseng or placebo if they were stable and had no COPD exacerbation during the 2-week run in period. Visits were scheduled for baseline, end of run-in (week 2), end of treatment (week 6) and end of follow-up (week 10) (Figure 1). In the full-scale trial participants will be enrolled for 52 weeks, four weeks run-in, 24 weeks treatment and 24 weeks follow-up (Figure 2). They will attend 6 visits: baseline, randomisation, mid-treatment, end of treatment, mid follow-up and end of follow-up.

Ethical approval (B2012-49-01) was obtained from the Guangdong Provincial Hospital of Chinese Medicine Human Research Ethics Committee (Additional files 1 and 2). The pilot trial has been registered with the Australian and New Zealand Clinical Trials Registry (ACTRN: 12614000029695).

\section{Random assignment and blinding}

A list of randomisation numbers was generated by a statistician using statistical software (SPSS, Windows Version 20.0; IBM Corp., Armonk, NY). This process tested the effectiveness of keeping participants, investigators, medical staff, and other staff blinded to the study allocation. Opaque envelopes containing a number concealed to the treatment allocation was used to randomly assign participants to either $P$. ginseng or placebo group. Medications were put in packages that concealed the randomization code and were dispensed from the hospital's central pharmacy by pharmacists who were blinded to allocation.

\section{Interventions}

Participants in the intervention group received standardised P. ginseng extract, G115, in capsule form $200 \mathrm{mg}$ twice daily and participants in the control group received placebo for 4 weeks. P. ginseng G115 is manufactured according to Good Manufacturing Practices by Ginsana SA, Switzerland. The lactose-based placebo was also manufactured by Ginsana SA and matched in 


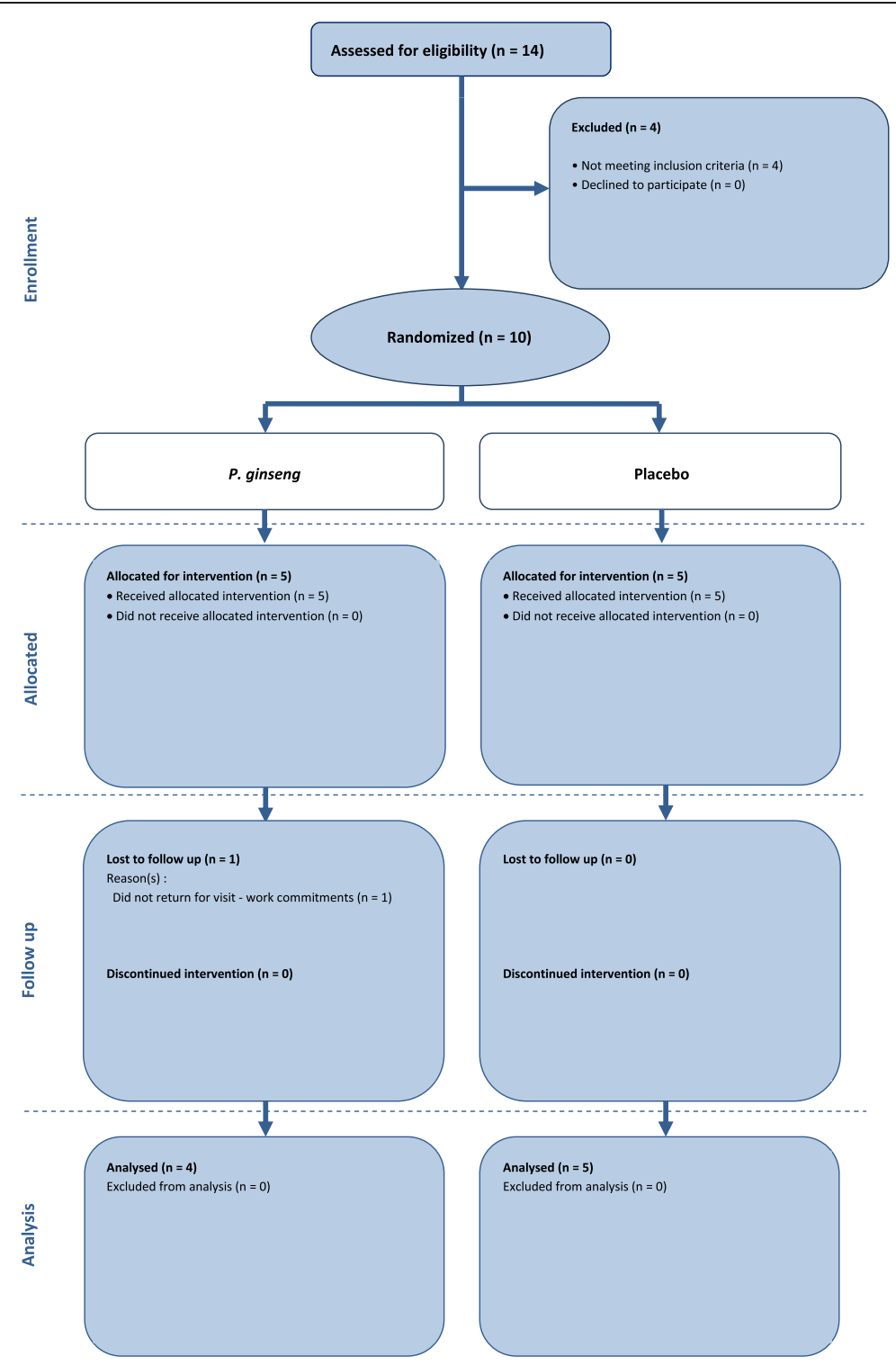

Figure 1 Flow chart describing study design and participant selection in the pilot trial.

appearance, taste and odour. In the full-scale trial medications would be dispensed for 24 weeks.

P. ginseng, G115, is the highest quality standardised extract and has been evaluated in more than 46 clinical studies over 35 years [12]. G115 is standardised to contain 4\% ginsenosides, Rg1, Rb1, Re, Rf, Rg2, Rc, Rb2, and $\mathrm{Rd}$. The dosage in this study was determined by referencing the clinical trial literature and recommendations from the manufacturer [13].

Throughout the study, participants were given the short-acting $\beta 2$-agonist Ventolin (salbutamol) to relieve symptoms as needed. Respiratory drugs, including longacting anticholinergics or long-acting $\beta 2$-agonists alone or in combination with glucocorticoids, could be used throughout the study under the advice of the participants' respiratory physicians. Other respiratory drugs, such as short-acting anticholinergics, short-acting $\beta 2$ agonists other than salbutamol, theophylline, corticosteroids as monotherapy, antibiotics, mucolytics and antitussives were not allowed during the study. The following medications for other conditions were also not allowed during the study: immunotherapy, monoamine oxidase inhibitor antidepressants, anticoagulants, antihyperglycaemics, and other Chinese herbal medicines.

\section{Outcome measures}

The primary outcome measure was rate of exacerbation, which was defined as a change in baseline dyspnoea, 


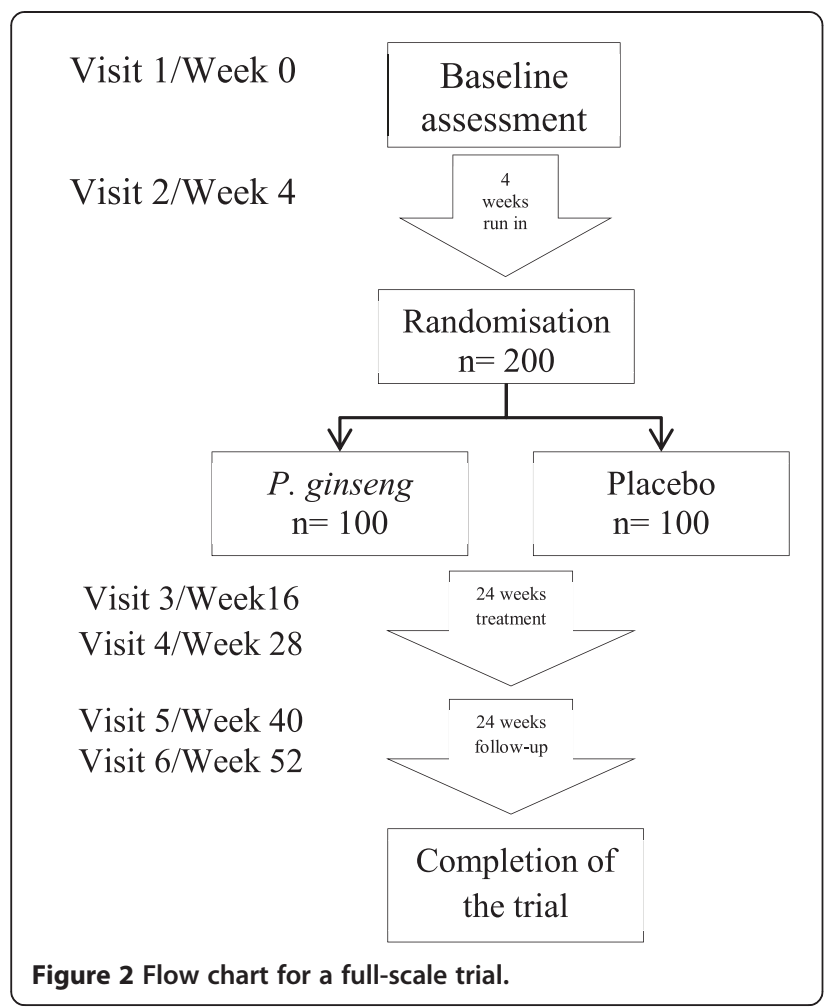

cough and/or sputum [14]. Specifically, exacerbations involving two or more symptoms, such as worsening dyspnoea and an increase in sputum purulence or volume or both, or any single major symptom and more than one minor symptom such as upper airway infection, unexpected fever or increased wheezing that lasted two or more days [14]. Investigators (who are qualified respiratory research assistants) phoned the participants at the end of every week and reviewed their participant diary at each visit to determine if a participant had experienced an exacerbation. Investigators prescribed medications to treat exacerbations if needed. Exacerbation severity was categorised as mild (easily tolerated by participant, causing minimal discomfort), moderate (discomfort significant enough to interfere with daily activities) or severe (incapacitating, unable to work or perform daily activities). Exacerbations were not considered adverse events unless they were serious (e.g., fatal, life threatening, permanently incapacitating or left participants needing prolonged hospitalisation).

Secondary outcomes were health status measured with the St. Georges Respiratory Questionnaire (SGRQ), COPD Assessment Test (CAT), the Short-form Health survey (SF-36) and exercise tolerance using the 6-minute walking test (6MWT). Other outcomes included change in postbronchodilator FEV1 and FVC, use of relief medication, and COPD-specific medical resource use, including emergency department presentations and medical practitioner visits. Safety assessments included investigator inquiries about the occurrence of adverse events and blood samples, which included a full blood count and liver/ kidney function tests taken before and after treatment.

All outcome measures were collected by the same investigator for continuity and to aid in maintaining a standard procedure. Outcome measures were selected based on the anticipated full-scale trial. For a full-scale trial the sample size and adequate statistical power would be based on a study evaluating carbocisteine for acute exacerbations of COPD [15].

\section{Statistical analysis}

Outcome measures were analysed by $t$ test at the end of each time point. SPSS, Windows Version 20.0 (IBM Corp., Armonk, NY) was used to analyse the data for the outcome measures. Last Observation Carried Forward (LOCF) was used to evaluate data with an intention-totreat analysis. Data were presented as mean and standard deviation. The aim of this pilot trial was to test the practicality of trial design, it would be inappropriate to make any inferences or state findings of any efficacy. Data were only analysed to make the investigators aware of any issues that needed to be overcome in the planned larger-scale trial.

\section{Results of the pilot trial}

We recruited 14 participants from a community health program at the Guangdong Provincial Hospital of Chinese Medicine in May and June of 2012. After the 2-week run in period, 10 of these participants were eligible for the study and were randomly assigned to $P$. ginseng group $(\mathrm{n}=5)$ (200 mg twice daily for four weeks) or placebo group $(\mathrm{n}=5)$ (Figure 1$)$. The four excluded participants did not meet the inclusion criteria due to their lung function and an exacerbation of COPD symptoms during the run-in period and abnormal liver function and a history of liver disease. The participants' mean age was 64.8 years (range 57 to 73 years old). Nine participants were male and one was female. One participant in the $P$. ginseng group dropped out at week three because of work commitments. Nine participants were classified as having severe COPD, one was classified as having moderate COPD and none were classified as having very severe COPD.

A qualified Chinese medicine respiratory specialist diagnosed the participants' health according to Chinese medicine. Seven participants were considered to have lung and kidney qi deficiency, and three participants were considered to have lung, spleen and kidney qi deficiency. At the start of the study, two participants were taking antibiotics, eight were taking long-acting $\beta$-agonists, and nine were taking inhaled corticosteroids. Because of the small sample size, several baseline factors, including smoking status and coughing severity, were imbalanced (Table 1). 
Table 1 Baseline characteristics of the participants

\begin{tabular}{|c|c|c|c|c|c|c|}
\hline \multirow{2}{*}{$\begin{array}{l}\text { Baseline assessment } \\
\text { Age (years) }\end{array}$} & \multicolumn{3}{|c|}{ Treatment group $(n=5)$} & \multicolumn{3}{|c|}{ Placebo group $(n=5)$} \\
\hline & \multicolumn{3}{|c|}{$67 \pm 4$} & \multicolumn{3}{|c|}{$62.6 \pm 4.15$} \\
\hline Male/Female & \multicolumn{3}{|l|}{$5 / 0$} & \multicolumn{3}{|l|}{$4 / 1$} \\
\hline \multicolumn{7}{|l|}{ Smoking status } \\
\hline Current smoker & \multicolumn{3}{|l|}{3} & \multicolumn{3}{|l|}{0} \\
\hline Former smoker & \multicolumn{3}{|l|}{1} & \multicolumn{3}{|l|}{5} \\
\hline Never smoked & \multicolumn{3}{|l|}{1} & \multicolumn{3}{|l|}{0} \\
\hline FEV1 (L) (post bronchodilator) & \multicolumn{3}{|c|}{$1.38 \pm 0.63$} & \multicolumn{3}{|c|}{$0.83 \pm 0.10$} \\
\hline FVC (L) (post bronchodilator) & \multicolumn{3}{|c|}{$2.79 \pm 0.59$} & \multicolumn{3}{|c|}{$2.33 \pm 0.36$} \\
\hline FEV1/FVC (post bronchodilator) & \multicolumn{3}{|c|}{$0.48 \pm 0.13$} & \multicolumn{3}{|c|}{$0.36 \pm 0.06$} \\
\hline \multicolumn{7}{|l|}{ COPD severity } \\
\hline Moderate & \multicolumn{3}{|l|}{1} & \multicolumn{3}{|l|}{0} \\
\hline Severe & \multicolumn{3}{|l|}{4} & \multicolumn{3}{|l|}{5} \\
\hline Symptom severity & Mild & Mod & Sev & Mild & Mod & Sev \\
\hline Shortness of breath & 3 & 2 & 0 & 3 & 2 & 0 \\
\hline Cough & 2 & 3 & 0 & 5 & 0 & 0 \\
\hline Sputum production & 3 & 1 & 1 & 5 & 0 & 0 \\
\hline Antibiotics & \multicolumn{3}{|l|}{0} & \multicolumn{3}{|l|}{2} \\
\hline Long-acting beta-agonists & \multicolumn{3}{|l|}{3} & \multicolumn{3}{|l|}{5} \\
\hline Inhaled corticosteroids & \multicolumn{3}{|l|}{3} & \multicolumn{3}{|l|}{5} \\
\hline \multicolumn{7}{|l|}{ Chinese medicine diagnosis } \\
\hline Lung and kidney qi deficiency & \multicolumn{3}{|l|}{4} & \multicolumn{3}{|l|}{3} \\
\hline Lung, spleen and kidney qi deficiency & 1 & & & 2 & & \\
\hline
\end{tabular}

None of the participants experienced an exacerbation during the short study duration (10 weeks). Health status questionnaires (SGRQ, SF-36, and CAT) were unchanged in both groups (see Table 2). All participants, except the one who dropped out, completed the questionnaires and the result was evaluated by LOCF. The baseline questionnaire scores, except those for the SF-36 questions 2 and $3 \mathrm{~b}, 3 \mathrm{c}$, and 3e, were balanced between groups. There was no significant difference in responses to the questionnaires between groups at the end of treatment (see Table 2).

Table 2 Outcome measures

\begin{tabular}{|c|c|c|c|c|c|}
\hline & & & & & \\
\hline & (mean \pm & d deviation) & (mean \pm & d deviation) & $P$ value^ \\
\hline & Baseline & End of treatment & Baseline & End of treatment & \\
\hline Exacerbations & Not applicable & 0 & Not applicable & 0 & 1 \\
\hline FEV1 (L) & $1.38 \pm 0.63$ & $1.40 \pm 0.60$ & $0.83 \pm 0.10$ & $0.80 \pm 0.06$ & 0.06 \\
\hline FVC (L) & $2.79 \pm 0.59$ & $3.00 \pm 0.50$ & $2.33 \pm 0.36$ & $2.40 \pm 0.46$ & 0.11 \\
\hline FEV1/FVC & $0.48 \pm 0.13$ & $0.40 \pm 0.10$ & $0.36 \pm 0.06$ & $0.30 \pm 0.06$ & 0.14 \\
\hline SGRQ & $37.20 \pm 12.85$ & $32.20 \pm 12.20$ & $45.80 \pm 10.54$ & $34.40 \pm 8.20$ & 0.74 \\
\hline CAT (total score) & $17.0 \pm 6.32$ & $16.20 \pm 5.40$ & $17.20 \pm 4.87$ & $14.40 \pm 2.70$ & 0.67 \\
\hline SF-36-General health & $40.0 \pm 23.18$ & $48.00 \pm 20.70$ & $37.0 \pm 14.40$ & $39.0 \pm 18.50$ & 0.49 \\
\hline SF-36-Mental health & $79.20 \pm 17.75$ & $82.40 \pm 12.10$ & $86.40 \pm 7.26$ & $89.60 \pm 6.60$ & 0.49 \\
\hline 6MWT (m) & $518.20 \pm 10.01$ & $502.80 \pm 29.0$ & $453.20 \pm 60.27$ & $453.0 \pm 20.50$ & $0.01^{*}$ \\
\hline
\end{tabular}

$\wedge$ End of treatment between groups comparison.

${ }^{*} P<0.05$ indicated significant difference between groups.

6MWT: 6-minute walking test, CAT: COPD Assessment Test, FEV1: forced expiratory volume in 1 second, FVC: forced vital capacity; SGRQ: St Georges Respiratory Questionnaire, SF-36: Short-form health survey (version 2). 
The distance walked in six minutes at the end of treatment was not clinically important and the absolute distance walked did not change. In the $P$. ginseng group the mean distance walked at baseline was 518.2 meters and at the end of treatment it was 502.8 meters. In the placebo group, the mean distance walked at baseline and at the end of treatment did not change, 453 meters. There was also no change in mean FEV1, FVC, or FEV1/FVC in the $P$. ginseng or placebo groups (Figure 3). All participants used relief medication (salbutamol) at some stage throughout the study. On average, participants in the $P$. ginseng group used $4.82 \pm 1.6$ puffs per week and participants in the placebo group used $2.06 \pm 1.7$ puffs per week. None of the participants needed to use any other COPD-related medical resources.

There were no adverse events recorded from the study medication. One participant in the $P$. ginseng group experienced a "common cold", with symptoms of a sore throat, cough and low-grade fever. This was not considered an exacerbation or related to the treatment. For the nine participants, blood haematology and biochemistry were unchanged after the treatment phase. The other participant showed a slight increase in white blood cell count and neutrophils. The result was not considered to be clinically important.

Potential participants were relatively easy to identify. The participants could not tell the difference between the $P$. ginseng treatment and placebo. Those given
P. ginseng tolerated it well. Participants did not report any issues in taking the dose twice a day for both groups and did not report that the capsules tasted badly or were hard to swallow. Despite multiple outcome measures, each visit at the hospital by the participants lasted for no more than 1.5-2 hours.

\section{Discussion}

This report presented a study protocol and the results of a pilot trial comparing $P$. ginseng with placebo for moderate to very severe COPD participants. The pilot trial was run without major issue according to the study protocol in a hospital environment in China.

The randomization process was successful and the use of opaque envelopes to conceal allocation was effective. The study procedures, including lung function, questionnaires, walking distance and blood tests were completed at all of the time points. Response rates for data collection were high; the only missing response was that of the participant who dropped out. No participants were excluded on a Chinese medicine diagnosis in the inclusion criteria.

Because of the small sample size and short study duration it was not surprising to observe no difference in outcomes. A larger trial with proper treatment duration according to the treatment principle would reveal the actual effect of $P$. ginseng in treating COPD. The planned full-scale trial protocol will be conducted over one year (6 months treatment and 6 months follow-up), similar to a

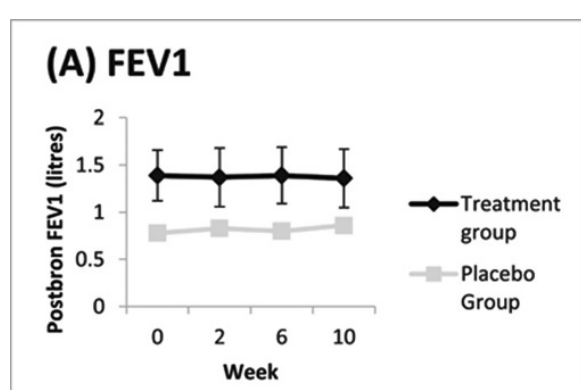

(B) FVC

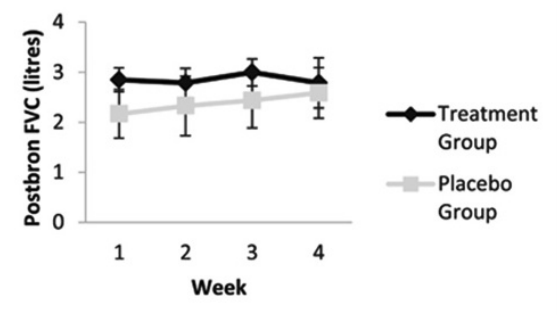

\section{(C) FEV1/FVC}

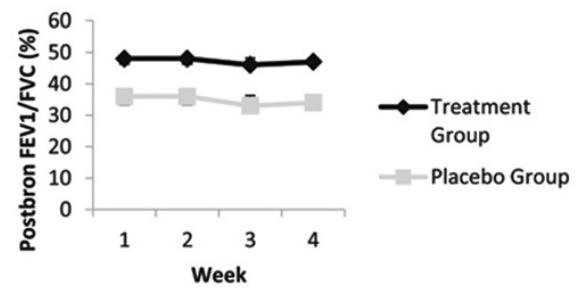

\section{(D) SGRQ}

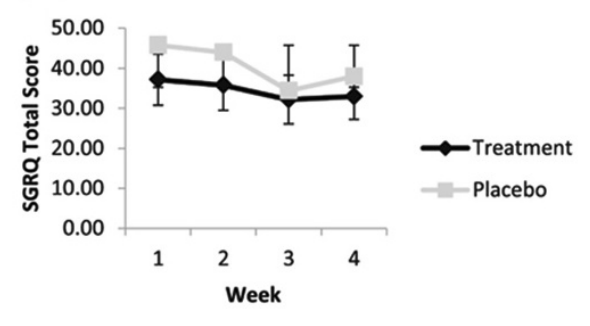

Figure 3 Lung function and SGRQ results: (A) mean post-bronchodilator FEV1 in litres, (B) mean post-bronchodilator FVC in liters, (C) post-bronchodilator FEV1/FVC percentage and (D) SGRQ total score. Error bars were standard deviations. There was no significant difference between groups at any time point. 
current Australian trial evaluating $P$. ginseng for moderate COPD [10].

A key consideration for any full-scale trials is recruitment. In this pilot study, four out of 14 participants (28\%) did not meet the inclusion criteria. Recruitment for a full-scale trial would succeed at a large hospital or multiple sites. One participant withdrew from this study. Participant withdrawals from long-term studies especially studies on COPD, are between $30 \%$ and 50\% [16].

The outcome assessment in the pilot trial by only one investigator performing all tests, including spirometry using one device at the same time of day (8 a.m.12 p.m.) might be logistically unrealistic in the full-scale trial. Therefore, standard operating procedures for all outcomes would be necessary. The definition of exacerbation severity should also be improved in a full-scale trial.

Based on the success of this pilot trial a full-scale trial has been implemented at the Guangdong Provincial Hospital of Chinese Medicine. The full-scale trial has received ethical approval and has been registered with the ANZCTR (ACTRN: 12613000382774). The sample size for that full-scale trial was calculated according to the effect size on rate of exacerbation in COPD patients using a mucolytic agent (carbocisteine) in a randomized controlled trial in China [15]. To potentially see a 30\% difference between the treatment and placebo with an $80 \%$ power and a two-tailed significance level of $5 \%$. It is estimated that a sample size of 100 participants per group would be adequate.

\section{Conclusions}

Participant recruitment, study design, data collection and outcome measurement have been tested in a pilot trial. A full-scale trial is warranted.

\section{Additional files}

Additional file 1: Human Research Ethics Committee approval.

Additional file 2: Participant consent form.

\begin{abstract}
Abbreviations
6MWT: 6-Minute walking test; ANZCTR: Australian and New Zealand clinical trials registry; CAT: COPD assessment test; COPD: Chronic obstructive pulmonary disease; FER: Forced expiratory ratio; FEV1: Forced expiratory volume in one second; FVC: Forced vital capacity; GOLD: Global initiative for chronic obstructive lung disease; LOCF: Last observation carried forward; PDE-4: Phosphodiesterase-4; RCT: Randomized clinical trial; SF-36: Short-form health survey; SGRQ: St. George's respiratory questionnaire.
\end{abstract}

\section{Competing interests}

The authors have no competing interests.

\section{Authors' contributions}

CCX, LL, ALZ, FCKT, CW, LW, and ZW conceived and designed the study protocol. LW, YC, XG, ZW conducted the pilot trial. LW, YMD, JLS, and YC collected data and wrote the manuscript. All authors read and approved the final manuscript.

\section{Acknowledgements}

This study was funded by the National Health and Medical Research Council (NHMRC) (Project Grant Number: 616609); an International Research Grant from the Guangdong Provincial Academy of Chinese Medical Sciences, China and a grant from the Science and Technology Planning Project of Guangdong Province (Project grant number: 2012B031800192). Trial medication and placebo were supplied free of charge by Ginsana SA, Switzerland. We thank Professor Xu Yinji and respiratory physicians at the Guangdong Provincial Hospital of Chinese Medicine for their help with recruiting participants. We also thank Drs Li Xiaoyan, Liu Shaonan and Cai Jianxiong who helped to implement the trial.

\section{Author details}

'Guangdong Provincial Hospital of Chinese Medicine, Guangdong 510120, China. ${ }^{2}$ Traditional and Complementary Medicine Program, School of Health Sciences and Health Innovations Research Institute (HIRi), RMIT University, PO Box 71, Bundoora, Victoria 3083, Australia. ${ }^{3}$ Department of Respiratory Medicine, Box Hill Hospital and Monash University, Box Hill, Victoria 3128, Australia. ${ }^{4}$ Department of Respiratory and Sleep Medicine, Austin Hospital, Heidelberg, Victoria 3081, Australia.

Received: 9 December 2013 Accepted: 11 August 2014 Published: 14 August 2014

\section{References}

1. Global strategy for the diagnosis, management and prevention of COPD - The Global Initiative for Chronic Obstructive Lung Disease (GOLD) 2014. http://goldcopd.org.

2. World Health Organization. http://www.who.int/respiratory/copd/en/.

3. Barnes PJ: New anti-inflammatory targets for chronic obstructive pulmonary disease. Nat Rev Drug Discov 2013, 12:543-559.

4. An X, Zhang AL, Yang AW, Lin L, Wu D, Guo X, Shergis JL, Thien FCK, Worsnop CJ, Xue CC: Oral ginseng formulae for stable chronic obstructive pulmonary disease: A systematic review. Respir Med 2011, 105:165-176

5. An X, Zhang AL, May BH, Lin L, Xu Y, Xue CC: Oral chinese herbal medicine for improvement of quality of life in patients with stable chronic obstructive pulmonary disease: a systematic review. J Altern Complem Med 2012, 18:1-14

6. Gross $D$, Krieger D, Efrat R: Ginseng extract $G 115^{\circ}$ for the treatment of chronic respiratory diseases. Scweiz Z Ganzheits Med 1995, 1:29-33.

7. Scaglione F, Weiser $\mathrm{K}$, Alessandria M: Effects of the standardised ginseng extract $\mathrm{G}_{115^{\oplus}}$ in patients with chronic bronchitis: A nonblinded, randomised, comparative pilot study. Clin Drug Investig 2001, 21:41-45.

8. Scaglione F, Cogo R, Cocuzza C, Arcidiacono M, Beretta A: Immunomodulatory effects of Panax ginseng C.A. Meyer (G115) on alveolar macrophages from patients suffering with chronic bronchitis. Int J Immunother 1994, 10:21-24.

9. Coon JT, Ernst E: Panax ginseng: a systematic review of adverse effects and drug interactions. Drug Saf 2002, 25:323-344.

10. Xue CC, Shergis IL, Zhang AL, Worsnop C, Fong H, Story D, Da Costa C, Thien FCK: Panax ginseng C.A Meyer root extract for moderate chronic obstructive pulmonary disease (COPD): study protocol for a randomised controlled trial. Trials 2011, 12:164.

11. Shergis JL, Parker S, Coyle ME, Zhang AL, Xue CC: Key considerations for conducting Chinese medicine clinical trials in hospitals. Chin Med 2013, 8:3.

12. Scaglione F, Pannacci M, Petrini O: The standardised G115 $15^{\circ}$ Panax ginseng C.A. Meyer extract: a review of its properties and usage. Evid Based Integ Med 2005, 2:195-206.

13. Shergis JL, Zhang AL, Zhou W, Xue CC: Panax ginseng in randomised controlled trials: a systematic review. Phytother Res 2013, 7:949-965.

14. Anthonisen NR, Manfreda J, Warren CPW: Antiobiotic therapy in exacerbations of chronic obstructive pulmonary disease. Ann Intern Med 1987, 106:196-204

15. Zheng JP, Kang J, Huang SG, Chen P, Yao WZ, Yang L, Bai CX, Wang CZ Wang C, Chen BY, Shi Y, Liu CT, Chen P, Li Q, Wang ZS, Huang YJ, Luo ZY, Chen FP, Yuan JZ, Yuan BT, Qian HP, Zhi RC, Zhong NS: Effect of carbocisteine on acute exacerbation of chronic obstructive pulmonary 
disease (PEACE Study): a randomised placebo-controlled study. Lancet 2008, 14:2013-2018.

16. Decramer M, Celli B, Tashkin DP, Pauwels RA, Burkhart D, Cassino C, Kesten S: Clinical trial design considerations in assessing long-term functional impacts of tiotropium in COPD: the UPLIFT trial. COPD 2004, 1:303-312.

doi:10.1186/1749-8546-9-20

Cite this article as: Wu et al:: Panax ginseng therapy for chronic obstructive pulmonary disease: a clinical trial protocol and pilot study. Chinese Medicine 2014 9:20.

\section{Submit your next manuscript to BioMed Central} and take full advantage of:

- Convenient online submission

- Thorough peer review

- No space constraints or color figure charges

- Immediate publication on acceptance

- Inclusion in PubMed, CAS, Scopus and Google Scholar

- Research which is freely available for redistribution 